\title{
Limit Strains for Severe Accident Conditions Applicable for Nuclear Power Plants
}

\section{Overview and Results of a Research Project of 13 European Institutions Sponsored by the European Commission}

\author{
R. Kieselbach \\ EMPA, Duebendorf, Switzerland \\ rolf.kieselbach@gmx.ch \\ R. Krieg \\ FZK, Karlsruhe, Germany
}

\section{SUMMARY}

The failure strains for a large variety of specimens of reactor pressure vessel material $22 \mathrm{NiMoCr} 37$ have been investigated. Most of the specimens contained holes or notches typical for a reactor vessel. Different load conditions and temperatures have been considered as expected in cases of severe accidents. The dependence of the failure strains on the size of the specimens was of special interest. This knowledge is needed to transfer results from small scale specimens and mock-ups to reactor dimensions.

Load versus load point displacement and hole or notch opening diagrams have been determined for the different specimen sizes. The evaluation of the results and the extensive post-test measurements of the crack surfaces of the broken specimens to get the very local failure strains are discussed.

Different theoretical models to predict failure strains were developed. Based on both experimental and theoretical findings, admissible strains for severe accident conditions can be proposed for the material. It is expected that using these limit strains in severe accident analysis, current over-conservatisms can be significantly reduced.

\section{INTRODUCTION TO OBJECTIVES OF LISSAC}

The current design rules for nuclear components allow only small structural deformations. They are appropriate for basic accident situations of the present design and rely on a stress-based concept. For extremely unlikely severe accidents, much larger plastic deformations with strains up to a certain fraction of 
the failure strain should be acceptable. In this case the analyses must use a more realistic strain-based concept. However, reliable information on local failure strains is lacking.

In particular, the influence of the component size on the failure strain is not known; but such information is indispensable if results from small scale specimens and model tests are to be applied to reactor dimensions.

Therefore, the research project LISSAC (Limit Strains for Severe Accident Conditions) has been launched to determine the failure strains and to propose admissible limit strains.

Based on the results from the present research program and from previous work, admissible strains will be proposed for different severe accident requirements. If, for instance, leakages must be avoided, the admissible strains will be moderate; if only the formation of missiles must be ruled out they can be larger.

\section{EXPERIMENTAL WORK}

\subsection{Work Program}

The main part of the experimental work consisted of several test families with different specimen shapes under uniaxial and biaxial static and dynamic loads at room temperature (RT), at $400{ }^{\circ} \mathrm{C}$ and at $850{ }^{\circ} \mathrm{C}$. Within one test family the specimen shapes and the other conditions were the same, but the size was varied up to reactor dimensions. Special attention was given to test families where the specimens have a hole or a notch causing non-uniform stress and strain distributions typical for local structural discontinuities in reactor vessel components. There are indications that for such non-uniform distributions size effects may be more pronounced than for uniform distributions. Thus, the investigations should allow to determine size effects on the failure strains and failure processes under conditions close to design configurations. As a basis, recent investigations on size effects of smooth specimens / $/$ / may be used. Related discussions and preliminary results of the current project may be found in $/ 2-6 /$. As the main result a proposal for admissible strains will be made.

The applicability of this result leading to a reduction of current over-conservatisms has been demonstrated for some selected severe accident problems. One important example is the corium slug impact against the pressure vessel head during a postulated in-vessel steam explosion. In the previous investigations $17,8 /$, only minor strains in the pressure vessel head were allowed and on this basis the load carrying capacity of the head in terms of kinetic slug energies was limited to $0.1,0.4$ and $0.8 \mathrm{GJ}$, depending on the conditions of the upper internal structures. As a result of the project LISSAC larger admissible strains in the head can be allowed. The increased load carrying capacity in terms of kinetic slug energies can be determined.

Another example might be the pressure vessel clamping which should withstand the dynamic loading caused by a postulated lower head failure, for instance. 


\subsection{Material}

The material for the specimens was taken from the cylindrical section of an unused reactor pressure vessel BIBLIS-C made from quenched and tempered material 22NiMoCr 37.

The locations of the different specimens within the cylindrical section of the available reactor pressure vessel were determined such that specimens belonging to the same test family were grouped together as closely as possible. In this way the influence of spatial variations of the material properties on the results within one test family could be minimized.

In addition, specimens with thicknesses much smaller than the wall thickness $t$ of the pressure vessel were located in the distance $t / 4$ from the outside surface of the reactor pressure vessel. Thus the reduced material strength usually found in the middle of the wall and the enhanced material strength at the surfaces of the wall did not appear in the smaller specimens; that means the influence of variations of the material properties through the wall thickness of the pressure vessel has been minimized too.

To get more specific answers about the mechanical material properties and their scatter, Charpy impact tests and standard tension tests have been performed for selected positions. Fig. 1 shows a typical variation of the material strength over the pressure vessel wall.

Since the carbon content is known to affect the mechanical material properties strongly, the spatial distribution of the carbon content in the cylindrical section of the reactor pressure vessel, where the specimens were made from, was investigated.

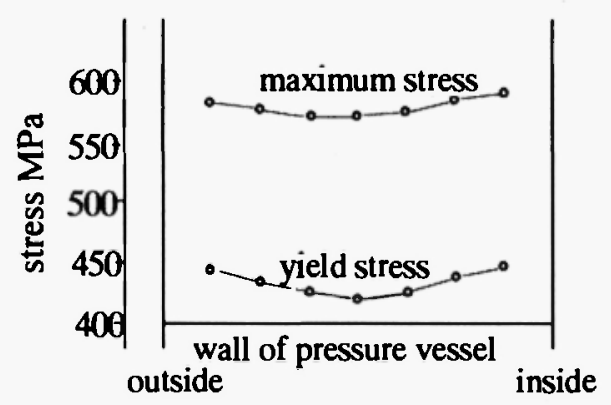

Fig. 1: Material strength over the pressure vessel wall (note the start of stress scale at $400 \mathrm{MPa}$ )

The variations over the cylindrical surface of the vessel were found to be rather small. The variations through the wall thickness were stronger.

From the results available it can be concluded that the scatter of the material properties is rather moderate. Its effect on the material strength within one test family is expected in the order of magnitude of $\pm 1 \%$; its effect on the material ductility is expected to be in the range of $\pm 5 \%$. 


\subsection{Specimens}

As mentioned before, several families of test specimens were designed to account for effects of size, stress concentration and biaxiality. The thickness (diameter) of the tensile and bend specimens varied between 4 (3) and $200(150) \mathrm{mm}$. Fig. 2 gives an overview over the different specimen types used. All specimens in different sizes were scaled.
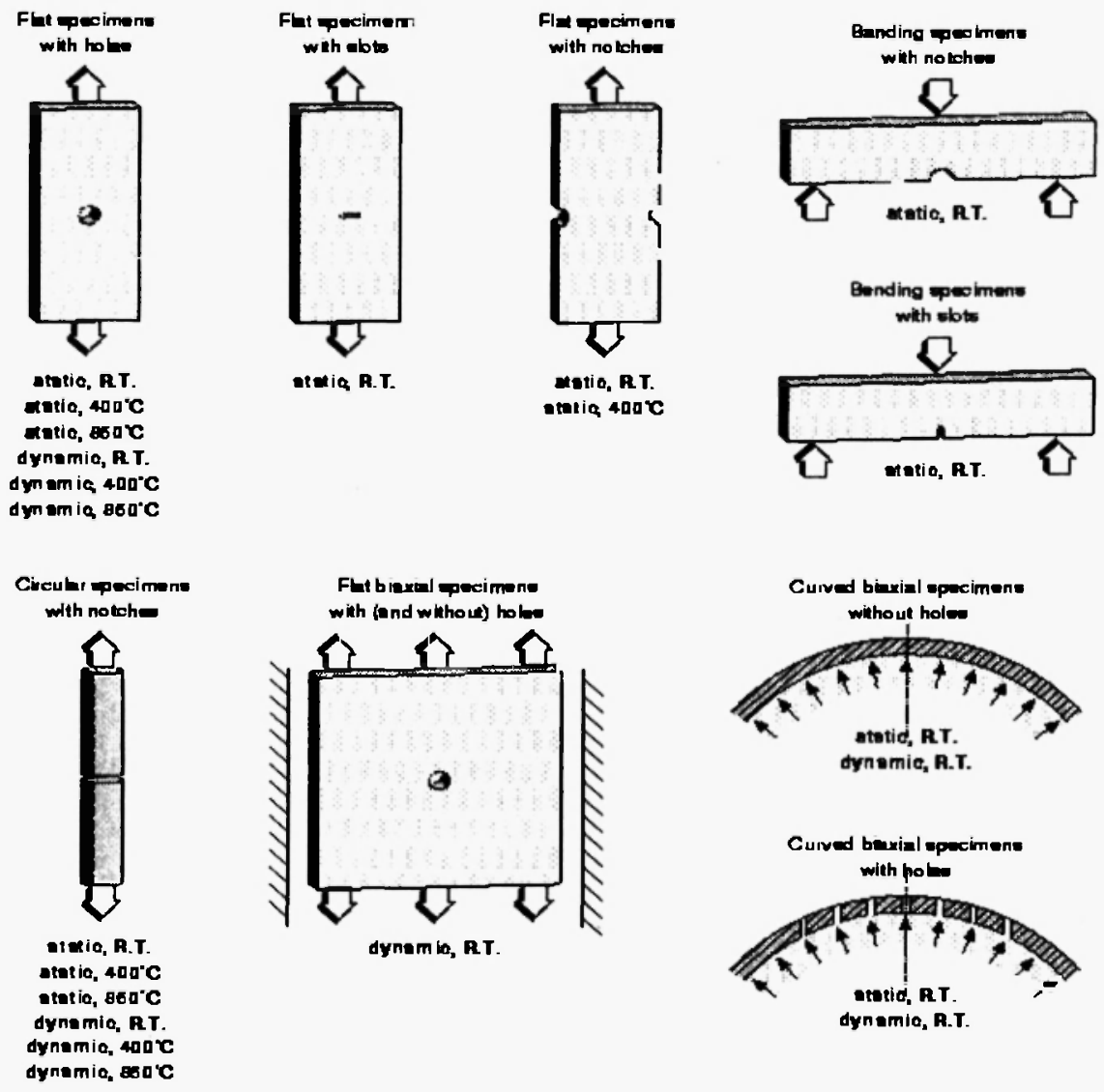

Fig. 2: The different specimen shapes under the listed load conditions define 22 test families

\section{TEST EQUIPMENT AND PROCEDURE}

For the specimens, which vary strongly in shape, load, temperature and size, very different testing machines were necessary. For the small tension tests standard machines were sufficient. However, the measurement of local maxima of strains required sophisticated methods such as the object raster technique. 
For the largest tension tests a very strong machine was needed as shown in Fig. 3. For dynamic tension tests with well defined strain rates a machine was used where the specimens were placed between very long prestressed steel cables. The dynamic load was provided by sudden release of the prestressing. Furthermore, a lot of additional test equipment and measuring techniques including micro-structural investigations were applied too.

Since single institutions own only one or two of these facilities and have only a part of the expertise needed, the formation of a consortium of different European institutions was a necessary prerequisite to perform the work.

If feasible, measuring grid lines were drawn on the surface of the specimens and/or reference points for displacement measurement were marked.

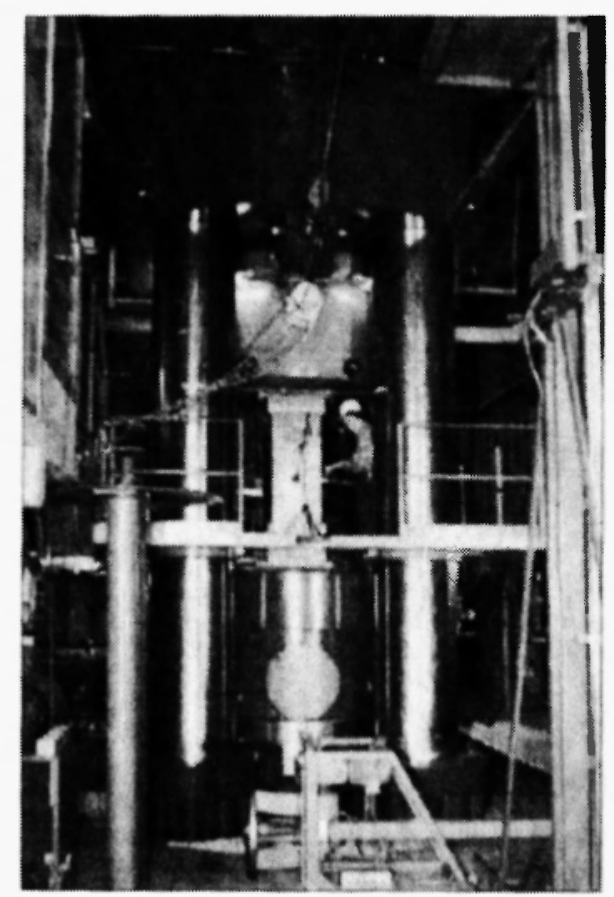

Fig. 3: $100 \mathrm{MN}$ Test machine at MPA-Stuttgart

(centre: tensile specimen ready for testing; right: note the size of the technician)

Heating rate and time were controlled as well as the attainment of the required temperature around the test section. Small specimens were heated in an inert atmosphere of argon gas to avoid development of scales.

Measurement of deformations was carried out either by direct observation of the reference points using a video-extensometer or for tests at elevated temperatures by attaching levers and rods, clamps and pin-points to the specimens, the displacement of which was then measured outside the temperature chamber, again by using the video-extensometer. 
Fig. 4 shows schematically how this worked and also the reference length of $6 t$ (6d) used for determination of overall strains.

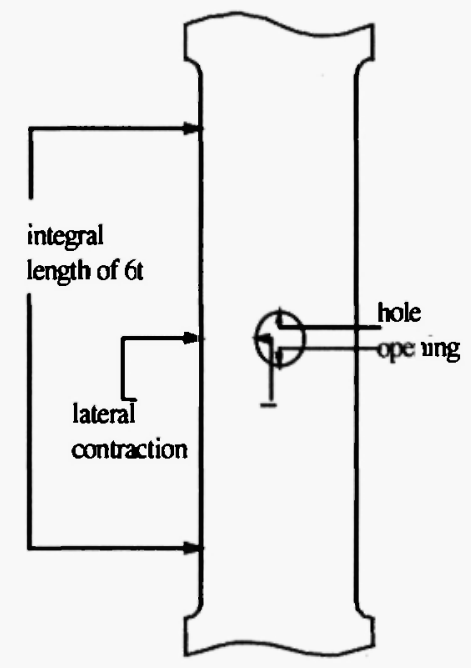

Fig. 4: Measurement of displacements, schematically

In Fig. 5 the reference points used for measurement of displacements in bend tests can be seen.

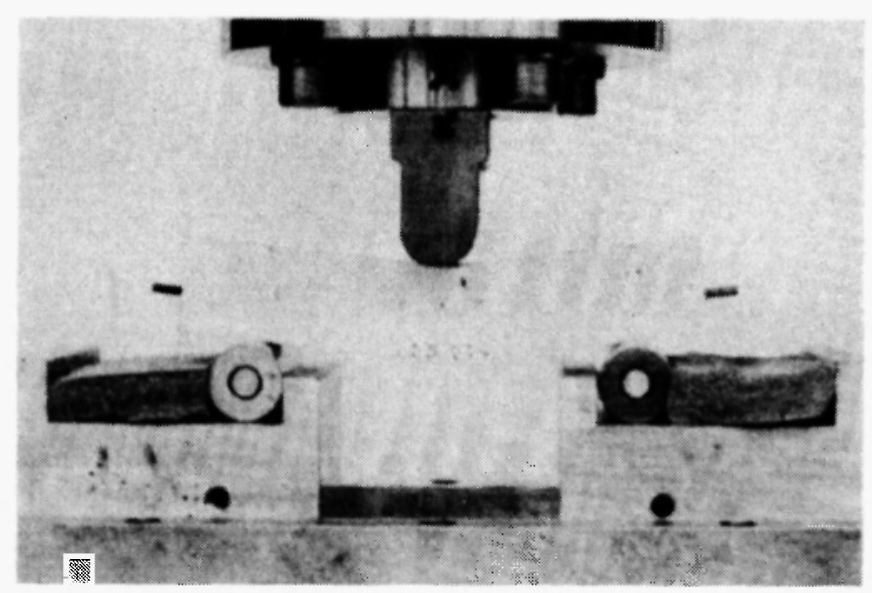

Fig. 5: Measurement grid painted on surface of bend specimen

Failure was assumed to have occurred when a crack had reached a detectable size. This size typically was less than $10 \%$ of the wall thickness or of the diameter of the specimen. The local failure strain was defined as the maximum equivalent strain reached at this instant before the crack causes major strain redistributions. 
The direct measurement of the local failure strain, i.e. the maximum equivalent strain at the instant when a crack can be just detected, is very difficult. For the LISSAC specimens with holes or notches, for instance, the maximum strain has a quite local character; it occurs at the curved surface of the hole or notch (inside the hole, for instance) and it reaches high values (often more than $100 \%$ ). Thus strain gauges can hardly be used. The application of markings and post-test measurements of the increased distances between these markings is only possible for large specimens. Also the application of a grid at the plane surface of a flat specimen with a hole or notches cannot really provide the maximum strain that occurs at the curved surface of the hole or notch, but for large specimens the grid can be used to determine the strain distribution close to maximum.

\section{METHODS TO DETERMINE THE LOCAL FAILURE STRAIN}

Because of the difficulties described above, indirect methods to determine the local failure strain have been developed within the LISSAC project. They are based on post test geometrical measurements on the broken specimens. The aim is to obtain a characteristic deformation $\delta$ of the specimen when a crack is just detectable. Based on this deformation the related strain fields in the specimen can then be reconstructed using theoretical models such as finite element models, for instance. For the different types of LISSAC specimens, appropriate indirect methods to determine the local failure strain were studied and applied.

For tension specimens with a hole or with notches the so-called "vanishing gap method" was applied. In Fig. 6 the method is illustrated for a flat specimen with a hole. The broken parts of the specimen are moved together such that the gap between the fracture surfaces vanishes. To reach this conditions most of the fracture zones of the broken specimen must overlap (Fig. $6 \mathrm{c}$ ). It is assumed that the configuration obtained in this way represents the shape of the specimen at the instant when the crack has started to develop and is just detectable. The location of this starting crack is the position where the fracture surfaces contact each other. It is well known that the starting crack will be always perpendicular to the tension stress (normal fracture). Therefore during the search for the position of the starting crack only elements of the fracture surfaces must be considered which are approximately perpendicular to the tension stress. The relative vertical hole increase, the so-called hole opening at this instant, is defined as the characteristic deformation $\delta$.

This reconstruction would be correct, if during the process of crack propagation the deformed surfaces of the hole did not suffer further shape changes. Although a precise proof of this hypothesis is not available yet, basic considerations of the stress redistribution due to the propagating crack (no tension stress normal to the crack surfaces) support the hypothesis strongly.

The major problem is that in reality the broken parts of the specimens cannot overlap (because two bodies cannot occupy the same space). Thus the configuration shown in Fig. $6 \mathrm{c}$ can be provided only virtually by computation. 


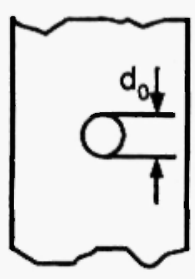

specimen before test

a

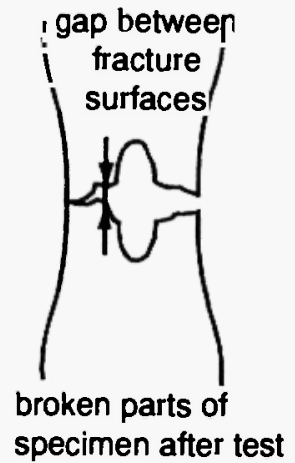

b

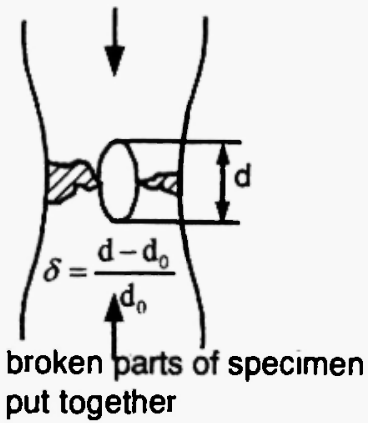

C

Fig. 6: Principle of vanishing gap method (the hatched parts in picture $c$ denote the fracture zone)

As indicated above, the relative hole or notch opening $\delta$ was used to reconstruct the complete strain fields in the specimen. For each family of tension tests one specimen was selected, the tension force increased monotonically step-by-step and finite element calculations were carried out for each step. To describe the material properties elastic-plastic stress-strain diagrams were used. Both, the strain fields and the hole or notch opening versus load were obtained. After the elimination of the load, the strain fields versus the hole or notch opening could be obtained. Thus the strain fields and especially the maximum equivalent strain, which represent the failure strain, are known for each hole or notch opening $\delta$ determined above.

Figure 7 shows the finite element models used.

For biaxial specimens other evaluation methods were used which are not described in this paper.

\section{SOME EXPERIMENTAL RESULTS}

In these results the tension forces versus the growing vertical diameter of the holes or the growing opening of the notches, Figs. 8 and 9 are shown. To allow a comparison of the results, the forces are divided by the smallest initial cross-sections of the specimens, which yields a nominal stress. If no size effect would occur, the curves for the tests belonging to the same family would be identical.

Especially the crack surfaces for the flat specimens with holes turned out to be quite irregular with large regions where shear stresses have caused failure. An example for such a crack surface is shown in Fig. 10. 

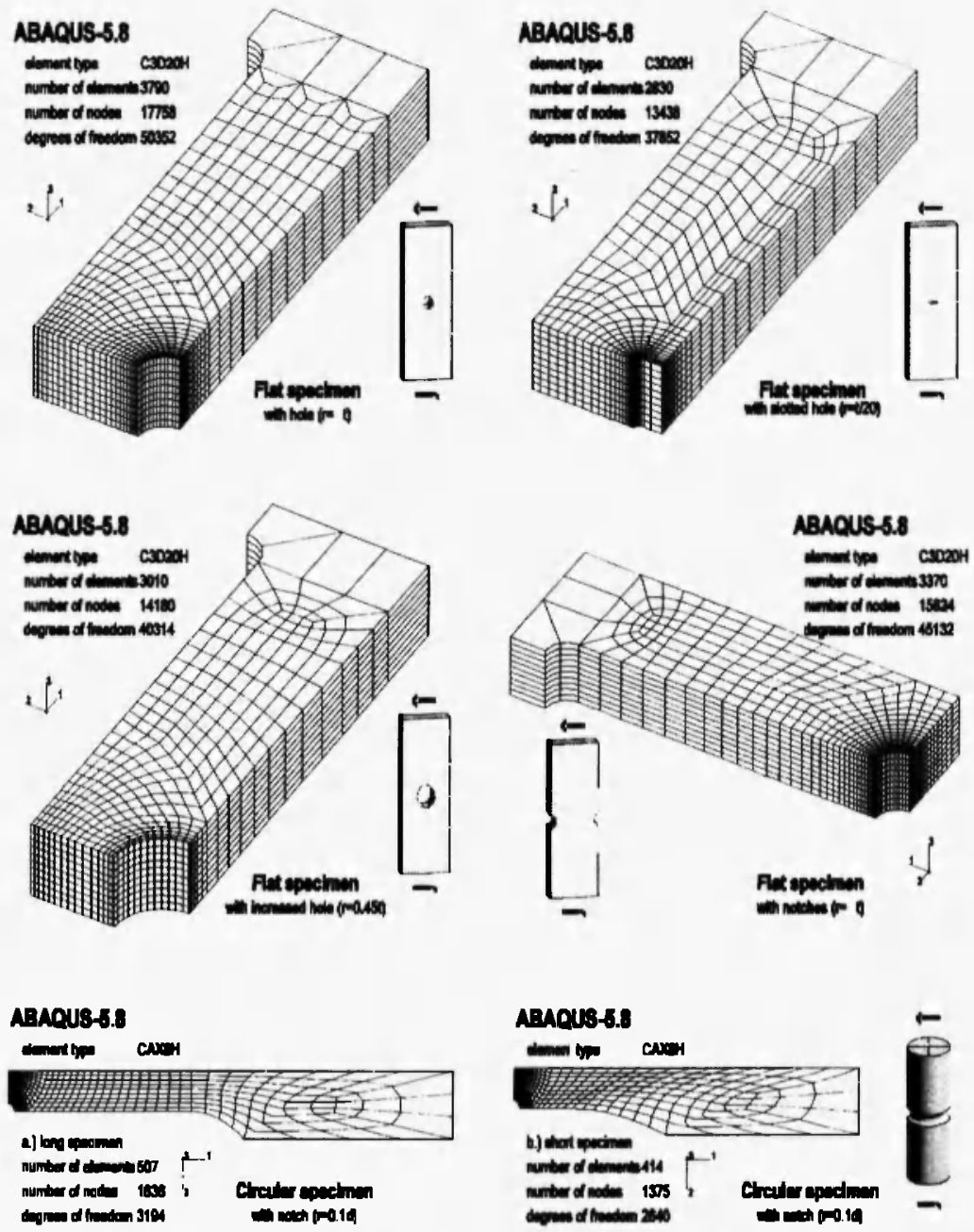

Fig. 7: Finite element models used to calculate the strain distributions.

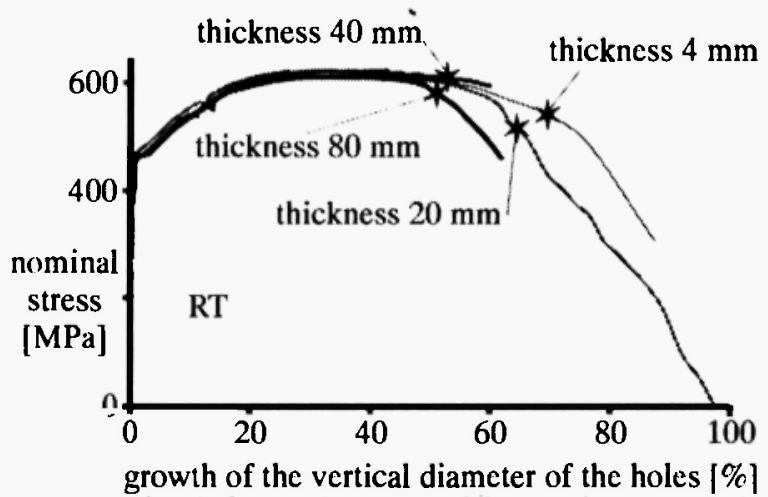

Fig. 8: Results for the test family of flat specimens with holes, static load, room temperature The stars mark the states where macroscopic cracks occur. 

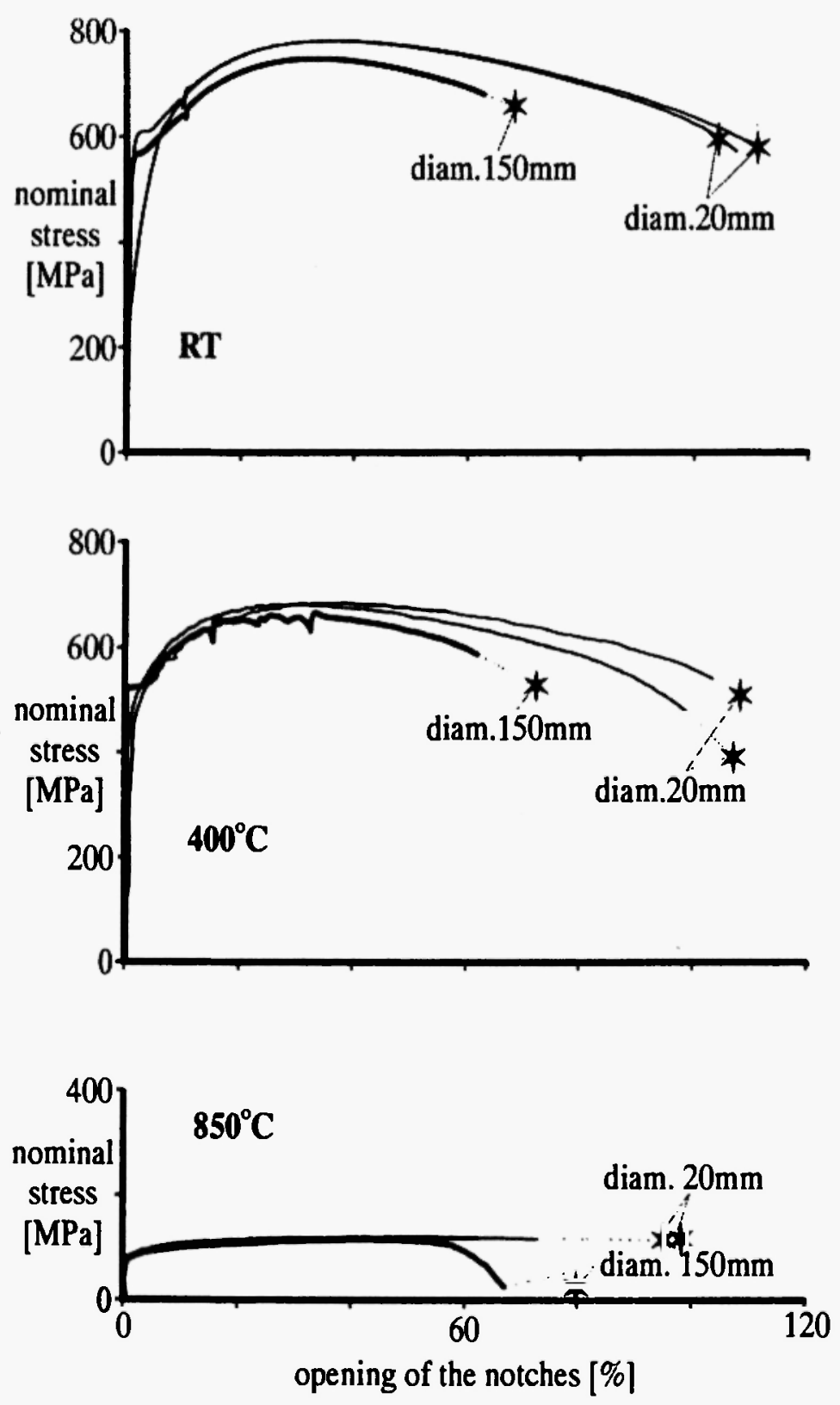

Fig. 9: Results for the test families of circular specimens with notches, static load, different temperatures; the stars mark the states where macroscopic cracks occur. 

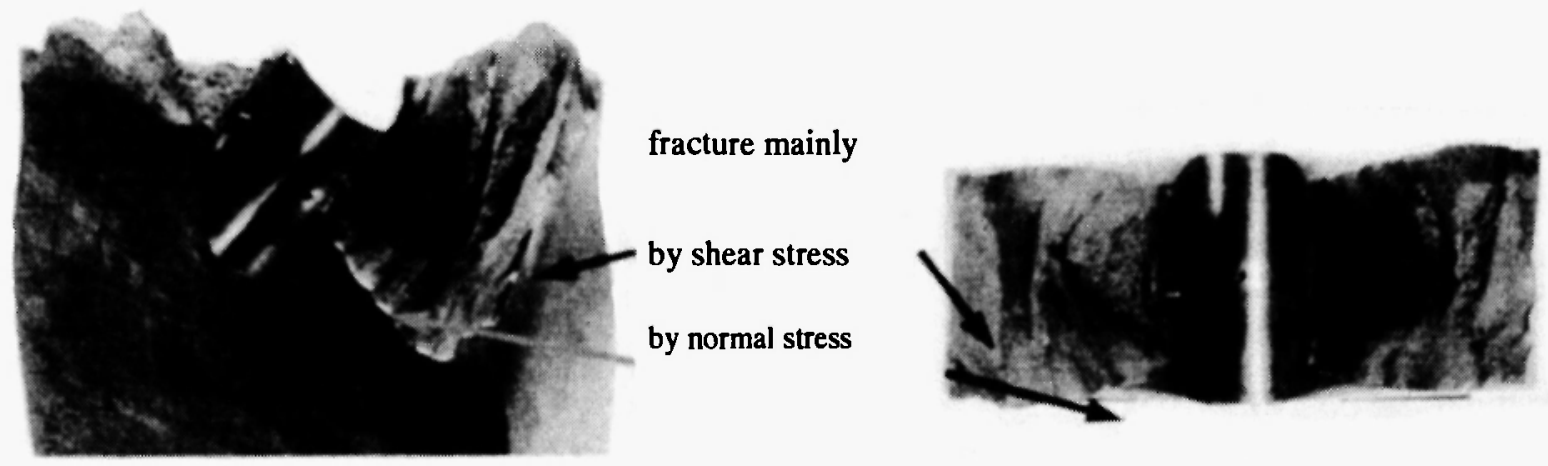

Fig. 10: Fracture surface of a broken flat specimen with a hole; left: perspective view, right: view in tension direction

\section{NUMERICAL MODELLING}

To deepen the understanding of structural degradation and of fracture and to allow extrapolations to other loading modes and geometrical shapes, advanced computational methods including damage models were developed. Since currently the physical phenomena affecting the failure strains are not very clear, quite different approaches were tried. In some cases so-called non-local concepts including gradient models and integral models, in other cases the description of stochastic properties at the grain size level were considered.

(A detailed report on the numerical modelling and implementation of models for damage into FEM-codes and of the results obtained will be given in separate publications.)

\subsection{Theoretical Models}

For the present problem the failure strains, i.e. the strains when a macroscopic crack starts to develop and the size dependency of these strains, are of main interest. This implies that the corresponding theoretical models will have to include both the phenomenon of damage and of a material related length - often called intrinsic length - affecting the deformation and damage process. The classical continuum material models for elasticity, plasticity or damage do not introduce such a length and even viscous or thermal effects do not suffice to reproduce the observed size effects. Therefore, an extension of the classical material models or the development of new models was necessary. Such models incidentally are also able to remove the so-called ill-posedness of deformation behavior emerging when the softening effects overrule the materials strainhardening. Actually, this ill-posedness means a malign mesh size dependency of the numerical results, which 
is unacceptable.

Quite different models have been studied in the past, but little was known about their applicability. Therefore in the LISSAC project several models and solution methods have been studied and were developed in parallel.

\subsection{Non-local Concepts}

Here the main activities were related to higher gradient models. First steps in this direction have been made within the REVISA project. Mostly these models extend the material law, e.g. the yield function or the evolution equation for internal variables by additive terms containing strain gradients. Two directions are followed in LISSAC. One is a more pragmatic way based on a Gurson-Tveergard damage model and a strain gradient extension proposed by Aifantis $/ 5 /$. The other way includes the development of a thermodynamically consistent micro-morphic approach. An implementation in the FEM code ABAQUS and first calculations of experiments revealing size effects and parameter fittings have already been done.

Mathematically closely related to these approaches are the integral concepts. Here the local values of some model variables are replaced by their spatial averages. The radius delimiting the domain for the spatial averaging can be correlated with the material intrinsic length. One easy use of this idea is the following. The critical state of a structure is reached when the damage, calculated in a conventional FEM analysis using a continuum damage model, exceeds a critical value in all points of the given domain and not only in one point.

Somehow related are the so-called advanced discrete modeling concepts. One partner has already implemented such a method. It uses the mesh size as the material intrinsic length, which is associated with the average distance of hard manganese sulphur inclusions. This procedure, combined with a Rousselier damage model, is able to model size scaled experiments even beyond the critical damage state in the domain of fracture. However, one major drawback of coupling the element sizes of the numerical scheme to microscopic quantities is that for large specimens a very large number of elements is needed.

\subsection{Stochastic Concepts}

In one approach a Voronoi tessellation method is used for a more direct modeling of the heterogeneous material at the meso-scale of grains. The individual grains are treated as continuous and are subdivided into finite elements. With the help of these simulations representative macro-models can be defined or statistical data on the mean values of the internal variables and on their temporal and spatial correlations can be derived. At present only two-dimensional simulations are possible.

With the help of the obtained statistical data a stochastic extension of the conventional flow function of a continuum damage model could be developed. The material intrinsic length enters the model via the spatial correlation of the white noise background field. Like with the weakest link theories, which were successfully 
applied for brittle materials, this more empirical approach should be able to model not only size effects, but also the experimentally observed scatter.

The results indicate, however, that stochastic models are hardly able to describe the experimental findings. Thus it may be concluded that stochastic phenomena do not essentially govern structural deformation and failure.

\section{MAIN RESULTS}

The experimental results show that within each test family either the maximum force of a specimen divided by the relevant cross section of this specimen or the maximum pressures applied at the specimen are approximately size independent. This also means that the loads scale in a very simple way, as expected, i.e. forces are proportional to the square of the length scale, pressures are scale invariant.

The results for the failure strains are more important. Some of them were not expected. The findings can be described as follows:

The local failure strains (logarithmic strains) are always higher than $50 \%$, which is much more than has been assumed so far in many analyses.

They decrease moderately with increasing specimen size, i.e. there is a moderate size effect. For the smallest specimens with thicknesses or diameters of only a few millimetres the local failure strains reach about $150 \%$; for very large tension specimens typical for a reactor pressure vessel the local failure strains decrease to about $70 \%$; for large biaxial specimens they decrease to 50 to $60 \%$.

One parameter which can describe the size effect is the radius of holes or notches located in the smallest cross section of the specimens where the stresses reach the highest values. The other dimensions of the specimens seem to play a minor role.

The strong influence of the radius of holes or notches on the local stress and strain has been well known for many years. However, the influence on the local failure strain is still unclear so far.

The type of specimens and load has only a small influence on the failure strain. Tests carried out with tension and bending specimens under uniaxal load, as well as tests with plate or shell type of specimens under biaxial load, give about the same failure strains - provided the hole or notch radius is the same.

Although this finding is in line with theory, it was not really expected when the project was started.

The above statements can be verified, if the failure strains are presented in diagrams like Fig. 11.

It includes all failure strains obtained at room temperature under static load. The different types of specimens and loading are indicated by different symbols. It turns out that the failure strains are indeed governed by the radius of the holes or notches, while the type of specimen and load has only minor influence. The somewhat smaller failure strains obtained for bending specimens might be caused by the visual inspection of these specimens during testing. So the crack could possibly be detected in an earlier state than with the vanishing gap methods. This seems to be especially true for the biggest bending specimen where the size of the detectable crack was very small in comparison to the notch radius. 


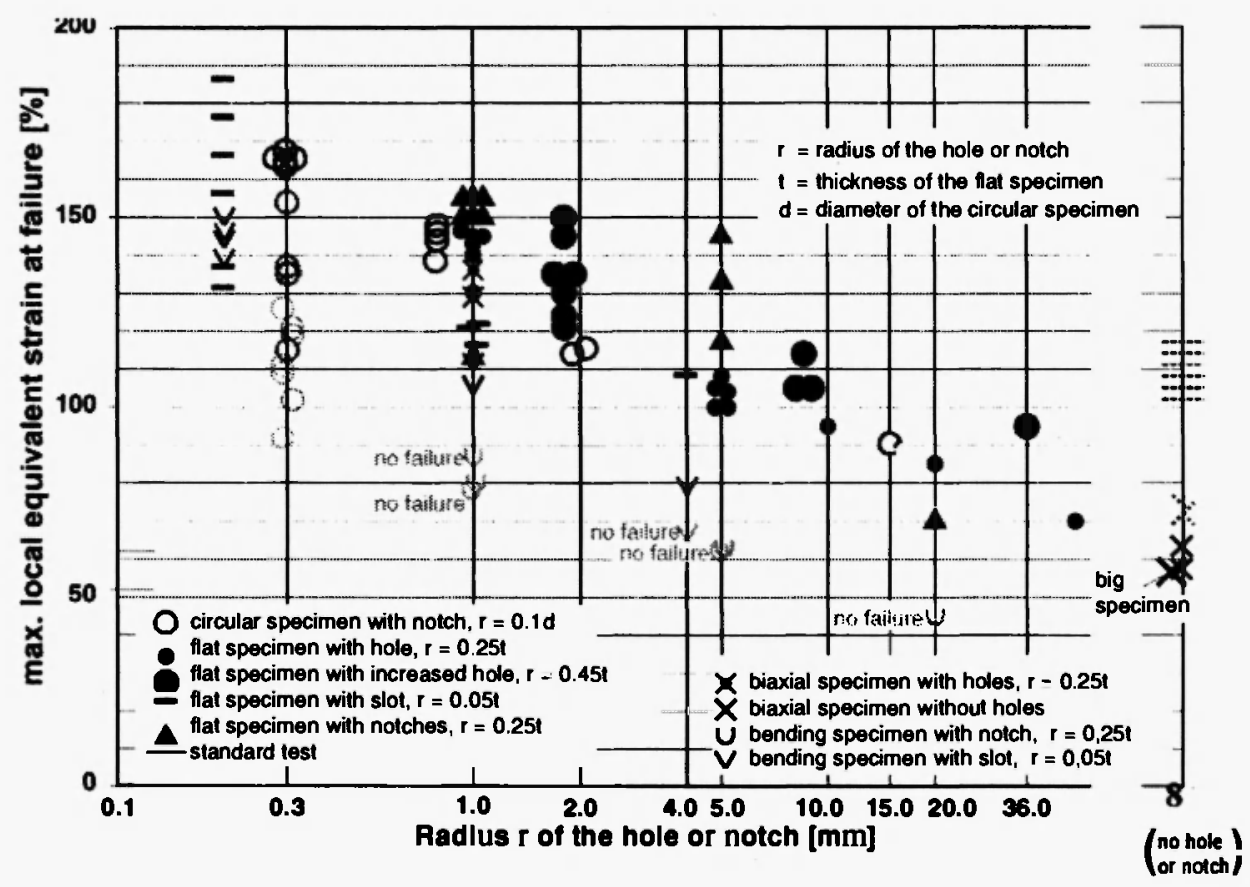

Fig. 11: Main results of LISSAC for RPV material at room temperature, static tests,

Specimens containing no holes or notches were interpreted as specimens with infinite holes or notches. This has been suggested by the gradient models, where the strain gradient vanishes for both specimens without holes or notches and for specimens with infinite holes or notches.

Note that for the biaxial specimens without holes or notches the failure strain does not depend on the specimen size. This is in line with the finding that the size effect on the failure strain is governed by the radius at the holes or notches.

The results obtained from the standard tension tests with smooth specimens (which do not belong to any test family) yield higher failure strains which do not fit with the general trend in Fig. 11. Perhaps here the radius should not be assumed to be infinite, but instead rather the radius of the necking before failure or even the radius of the cross section should be taken as the governing parameter.Here further studies would be welcome.

\section{PROPOSAL OF LIMIT STRAINS}

Since the ultimate goal of the project was the determination of permissible or limiting failure strains, a lower limit has to be determined from the test data. From Fig. 11 it can be seen that a clear trend exists for 
the failure strains and that this trend could easily be expressed by a mean curve or in the semilogarithmic presentation as a 50\% line. From a safety point of view confidence curves would be of interest showing the strains where for instance only $10^{-4}$ or even $10^{-6}$ of all the tested specimens are expected to fail. However to obtain such confidence curves just on the basis of statistical evaluations, a huge number of tests would be required.

This problem of a lower bound can also be tackled by relying to a larger extent on physical facts.
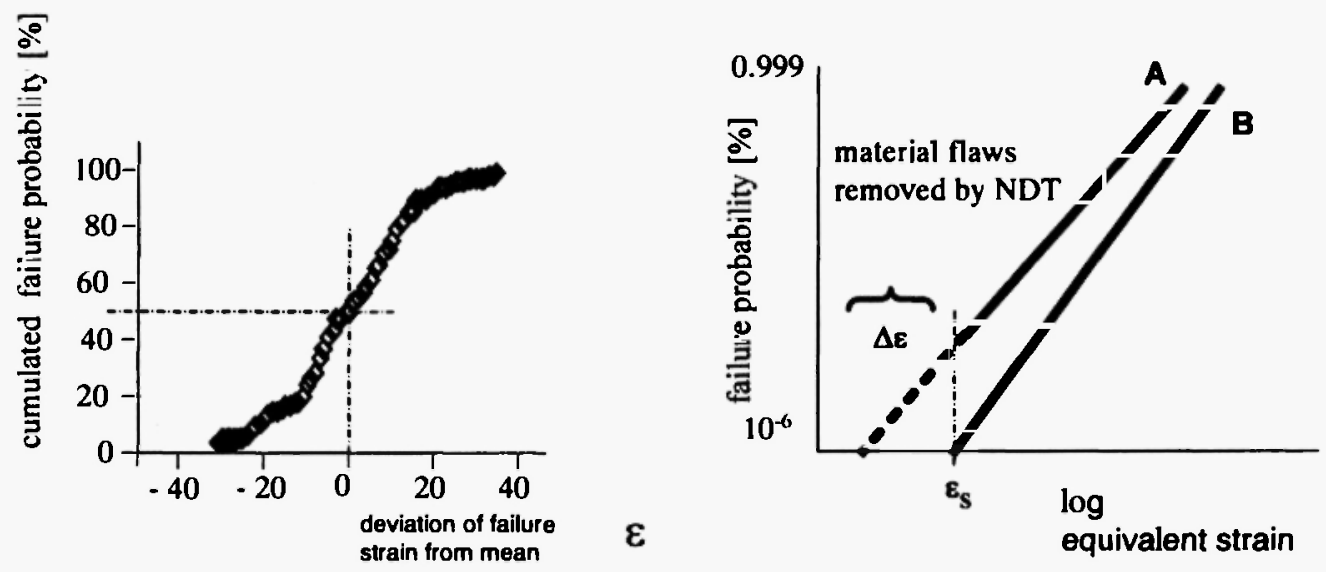

Fig. 12: Reduction of failure probability after removal of flaws by NDT

The steel is delivered with a certain, low probability of failure at quite moderate strains as shown by curve A of Fig. 12. The physical reason for such failures often are larger material defects like inclusions, voids, cracks, etc. If now non-destructive material tests are performed by which the larger material defects are detected, the material (and specimens) with such defects are excluded, failure at strains lower than a certain strain threshold $\varepsilon_{\mathrm{s}}$ will no longer occur. Consequently the probability of failure will now be described by curve $B$ of Fig. 12, which starts at the threshold $\varepsilon_{\mathrm{s}}$. If this threshold $\varepsilon_{\mathrm{s}}$ were known as a function of the hole or notch radius $r$, the confidence curve for strains where no specimens fail could be determined, provided, of course, that the non-destructive material tests were perfect.

The experimental results obtained suggest that

$$
\varepsilon_{\mathrm{s}}=\varepsilon_{\mathrm{o}}-\Delta \varepsilon,
$$

where $\varepsilon_{0}$ is the best estimate failure strain described by the straight blue line introduced in Fig. 13 and $\Delta \varepsilon$ is the deviation of the threshold from the best estimate failure strain. Since $\Delta \varepsilon$ can be assumed to be constant, the strain threshold $\varepsilon_{\mathrm{s}}$ is also a straight line. In Fig. 13 it is called the confidence curve. 


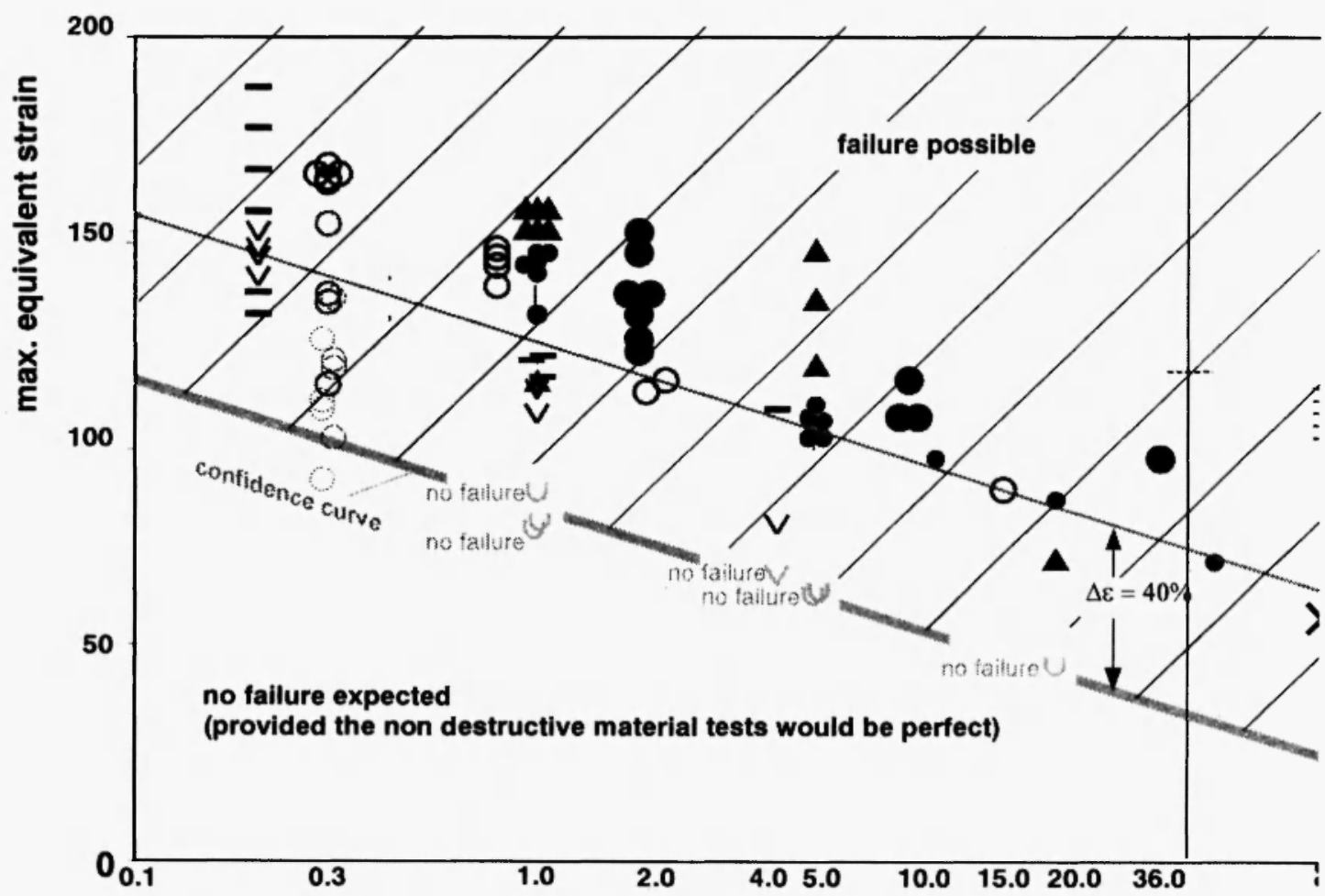

Fig. 13: No failure region for RPV material at room temperature and at $400^{\circ} \mathrm{C}$

It should be pointed out that below the confidence curve in Fig. 13 no failure is to be expected, if by nondestructive material tests the occurrence of large material inclusions, cracks, etc. could be absolutely prevented. In other words, below the confidence curve the probability of failure is given by the probability of large material defects that might remain undetected.

By similar considerations it was found that the failure strains obtained under dynamic loading at room temperature and at $400{ }^{\circ} \mathrm{C}$ and the related confidence curves may also be represented by the same straight lines discussed previously. This means that in the low and medium temperature region the local failure strains do not greatly depend on dynamic effects. Of special interest are the results obtained for biaxial specimens without holes under dynamic load: Again it turns out that the results do not depend on the specimen size, which supports the finding that the size effect is only governed by the hole or notch radius.

Of course, the results obtained at $850^{\circ} \mathrm{C}$ under static load shown in Fig. 9 are so different, that they can hardly be described by the straight lines applicable for specimens at room temperature and at $400^{\circ} \mathrm{C}$.

For $850^{\circ} \mathrm{C}$ and static load the failure strain seems to be about the same as for room temperature and 400 ${ }^{\circ} \mathrm{C}$, if only large notch or hole radii are considered. However the increase of the local failure strain with decreasing radius is much lower than in the cases of room temperature and $400{ }^{\circ} \mathrm{C}$. In other words, for 850 ${ }^{\circ} \mathrm{C}$ and static load there is almost no size effect on the failure strain visible. 
For $850^{\circ} \mathrm{C}$ and dynamic load the failure strains are generally higher than discussed before; but again, there is almost no size effect. Here it should be pointed out, however, that the data basis is rather poor and therefore no final conclusions should be made yet.

\section{CONCLUSIONS, BENEFIT OF THE PROJECT, LIMITATIONS}

The experiments carried out within the LISSAC project show that the local failure strain defined above as an equivalent strain (second invariant of the strain tensor) is almost independent of the shape of the specimen. This means that failure is mainly controlled by the equivalent strain. Other quantities characterizing the strain field (other invariants of the strain tensor, for instance) do have a significant influence. However, it should be pointed out, that for specimens under almost uniform three-axial tension stress (high three-axiality factor), the mean value of the tension stress can be expected to gain more influence and the failure strain can be expected to reduce considerably. Fortunately such cases hardly ever occur either in the specimens considered here or in reactor pressure vessels.

First results from the test family with flat tension specimens with a hole and from the test family of circular tension specimens with a notch show that the stress-deformation relations are not significantly effected by the specimen size. Only the deformations where macroscopic cracks occur decrease moderately with increasing specimen size. This seems to be true not only at room temperature, but also at elevated temperatures of $400^{\circ} \mathrm{C}$ and $850^{\circ} \mathrm{C}$.

The "so-called vanishing gap procedure" shows how to determine the characteristic local deformations (increased vertical diameter of the holes, opening of the notches) where macroscopic cracks occur. It shows, furthermore, how to determine the very local strain fields belonging to these deformations. The maximum values of these strain fields are defined as the failure strains. They decrease with increasing specimen size, just as the deformations where macroscopic cracks occur decrease with increasing specimen size.

Finally, the confidence curves introduced in section 9 are a certain fraction of the failure strains and therefore depend both on specimen size and confidence curves.

As mentioned before, the use of the proposed failure strains in severe accident analyses can be expected to decrease the current over-conservatisms significantly. That means that either the safety margins are higher than assumed in the past; or postulated accidents which are very severe (but extremely unlikely) can be shown to be sustained by the structures; or certain (proposed) safety measures can be shown to be unnecessary.

\section{ACKNOWLEDGEMENTS}

The sponsoring and financial support of this project by the European Commission under contract number 
FIKS-CT 1999-00012 is gratefully acknowledged.

The persons in charge at the institutions performing the project are: E. Aifantis (University of Thessaloniki), S. Bhandari and P. Benhamou (Framatome ANP, Paris), C. Caroli (ENEA, Roma), L. Cizelj (Institut Jozef Stefan, Ljubljana), J. Fokkens (NRC, Petten), P. Julisch and M. Seidenfuss (MPA, Stuttgart), D. Kalkhof (PSI, Villigen), R. Kieselbach (EMPA, Duebendorf), R. Krieg (FZK, Karlsruhe), K. Schramm (Framatome ANP, Erlangen), G. Solomos (EC-JRC, Ispra), H. Talja (VTT, Espoo), P. Verón (ENSA, Maliano).

Thanks to their combined efforts and contributions this project was successful.

\section{REFERENCES}

1. J. Devos et al., "Reactor vessel integrity in severe accidents" (REVISA), FISA 99, Luxembourg, Nov./Dec. 1999, 256-265.

2. T. Malmberg, K. Krompholz, G. Solomos and E.C. Aifantis, "Size Effects in Deformation and Fracture of a Ferritic Reactor Pressure Vessel Steel", SMiRT-16, Washington 2001, division F.

3. T. Jordan and T. Malmberg, "Failure Strain of the Reactor Pressure Vessel Head under Impact Loading", SMiRT-15, Seoul 1999, division L.

4. T. Malmberg, K. Krompholz, G. Solomos and E.C. Aifantis, "Investigations on Size Effects in Ferritic and Austenitic Materials", SMiRT-15, Seoul 1999, division L.

5. T. Malmberg, I. Tsagrakis, I. Elefteriadis and E.C. Aifantis, "On the Gradient Plasticity Approach to Size Effects, Part I: Reviews", Forschungszentrum Karlsruhe, Scientific Report FZKA 6321, Dec. 2000.

6. J. Aktaa, H.J. Hädrich, P. Julisch, M. Klotz and R. Schmitt, "Investigations to Size Effects on Plastic Deformation and Failure Behavior of In-homogenously Loaded Structures", SMiRT-16, Washington 2001, division $F$.

7. R. Krieg et al., "On the prediction of the reactor vessel integrity under severe accident loadings (RPVSA)", FISA 99, Luxembourg, Nov./Dec. 1999, 247-255.

8. R. Krieg et al., "Load carrying capacity of a reactor vessel head under a corium slug impact from a postulated in-vessel steam explosion",-Nucl. Eng. Des., 202, 179-196 (2000). 